\section{The injured snowman}

\author{
N Mahomed, MB BCh, FCRAD (D) \\ M Goodier, $M B C h B$ \\ M Modi, MB BCh, FCRad (SA), MMed \\ S Andronikou, MB BCh, FCRad, FRCR (Lond), PhD \\ Department of Radiology, University of the Witwatersrand, Johannesburg
}

\section{Abstract}

Total anomalous pulmonary venous return (TAPVR) is a rare congenital cardiac defect that may present with cyanosis, cardiac failure and failure to thrive in infancy. TAPVR type 1 has a typical radiographic appearance of the heart termed the snowman. We present a case of an infant with TAPVR type 1 with injuries in keeping with non-accidental injury (NAI) to highlight that children with chronic diseases are at greater risk of this kind of injury and that radiographic evaluation of the chest in cardiac disease plays a role beyond demonstrating the heart and vasculature of the chest.

\section{Case report}

A 2-month-old boy presented to the paediatric casualty unit with a fluctuating level of consciousness after allegedly falling from a bed. A CT brain scan (Figs 1 and 2) demonstrated a subarachnoid bleed associated with a right linear parietal skull fracture and an overlying scalp haematoma.

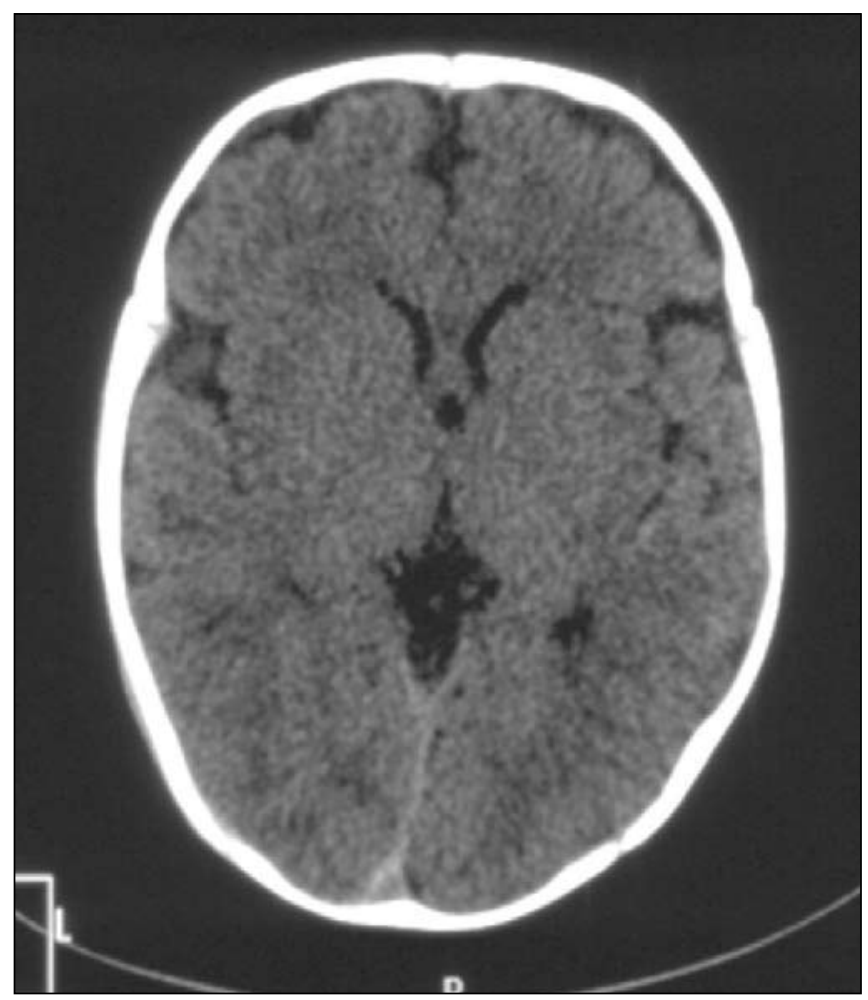

Fig.1. Non-contrast CT of the brain at the level of the Sylvian fissures demonstrates a subarachnoid bleed with the pseudo-delta sign.
On admission to the paediatric ward, hospital records identified the patient as belonging to the cardiac clinic for a cyanotic heart disease with a diagnosis of total anomalous pulmonary venous return (TAPVR) type 1 . Further investigation into the history revealed inconsistencies with the mechanism of injury and the suspicion of non-accidental injury (NAI) arose. A full skeletal survey was done. A chest X-ray (Fig. 3) demonstrated the classic snowman sign, in keeping with TAPVR type 1 . The chest X-ray also showed multiple, predominantly left-sided, posterior and lateral rib fractures with callus formation. No long bone fractures were identified.

\section{Discussion}

Our patient demonstrated radiographic features of TAPVR (the snowman sign) and of NAI. TAPVR results from the failure of pulmonary veins to connect to the left atrium. The pulmonary veins make an abnormal connection to the right atrium, with coronary sinus or systemic veins resulting physiologically in an extracardiac left-to-right shunt. This anomaly is accompanied by an intracardiac right-to-left shunt through a patent foramen ovale or an atrial septal

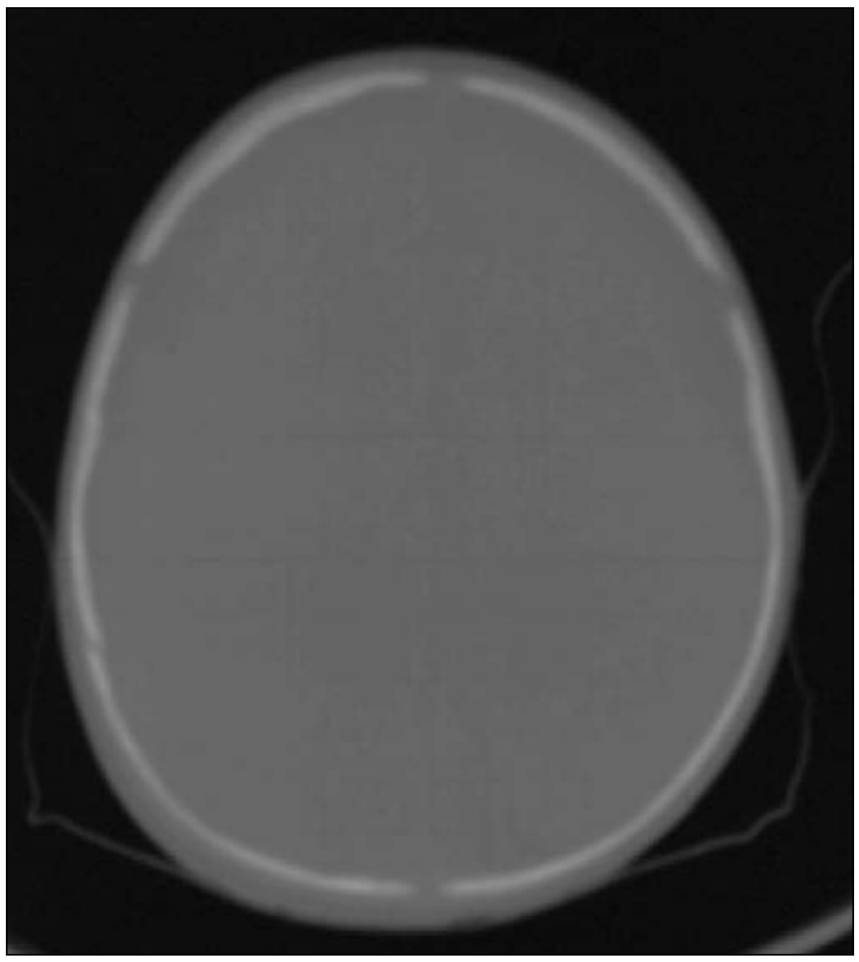

Fig. 2. CT of the brain with bone windows demonstrates a linear right parietal skull fracture. 


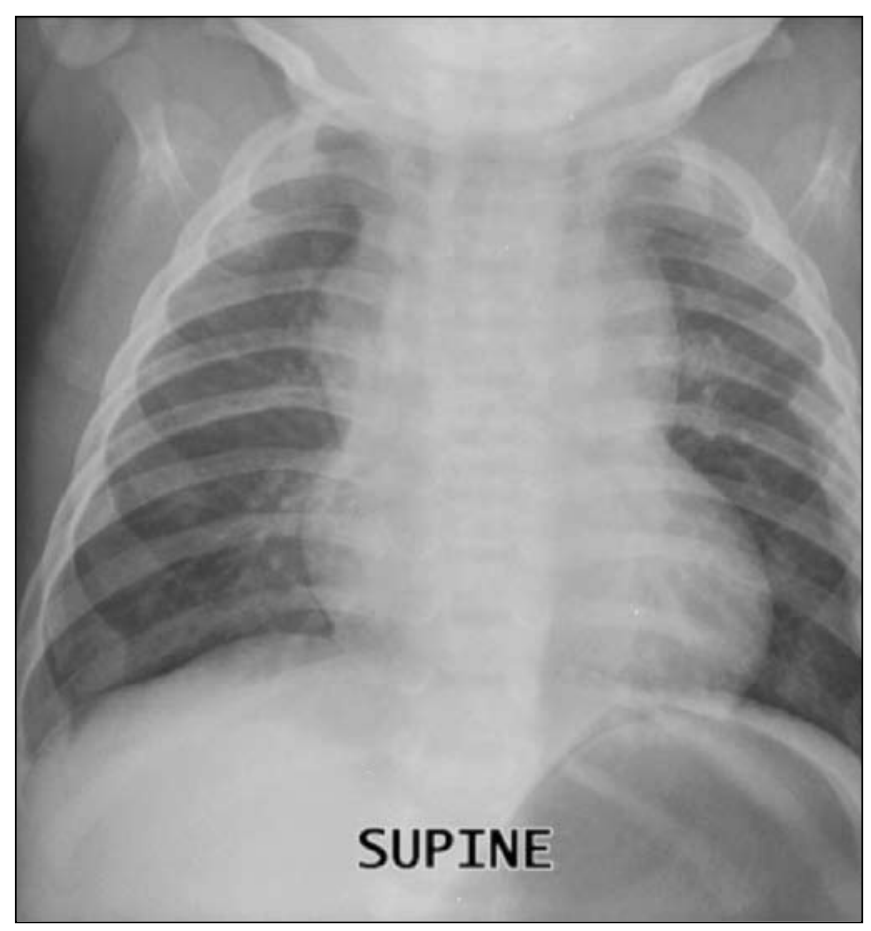

Fig. 3. Frontal chest $X$-ray demonstrating the classic snowman sign, in keeping with TAPVR type1 and multiple, predominantly left-sided, posterior and lateral rib fractures with callus formation.

defect. ${ }^{1}$ Clinical and radiological features depend on the location of the anomalous pulmonary venous connection. In TAPVR type 1 , the abnormal common pulmonary veins converge behind the heart to form the common pulmonary vein that connects to the vertical vein which drains in the left brachiocephalic vein. This results in the characteristic snowman appearance on chest X-ray. The head of the snowman is formed by the dilated vertical vein on the left, the superior vena cava on the right and the left brachiocephalic vein on top, while the body of the snowman is formed by the enlarged right atrium. ${ }^{1}$

Plasticity of the infant's skeleton and rib cage allows skeletal structures to deform rather than break until a threshold is reached. In infants without metabolic bone disease, rib fractures strongly correlate with NAI because the mechanism that generates these fractures is specific. ${ }^{2,3}$ Tight squeezing of the immature infant's chest results in a complex array of compressive and levering forces resulting in fractures of the posterior, lateral and anterior ribs. The pattern of rib fractures depends on the forces distributed in an area dependent on the size of the perpetrator's hands, and can be seen in similar locations in multiple adjacent ribs. ${ }^{2}$

In view of the underlying co-morbidity of a congenital cyanotic heart disease described in our patient, viewing the chest X-ray in isolation could result in misinterpretation of the multiple rib fractures as caused by cardiopulmonary resuscitation (CPR). Use of back supports previously used in CPR, limited the excursion of the posterior rib arc relative to the spine, therefore limiting levering of the posterior rib head over the transverse process and preventing posterior rib fractures. However, the recent development of the two-handed infant CPR technique allows the hands to encircle the chest similarly to how a perpetrator holds a child when shaking him. Therefore, posterior rib fractures, though uncommon, can occur with CPR. ${ }^{3}$ According to our patient's medical records, there was no history of CPR.

Non-accidental head injury (NAHI) occurs in approximately $12 \%$ of cases of physical abuse in children. ${ }^{2}$ In the absence of significant trauma, a skull fracture in an infant, as in our patient, suggests a higher likelihood of NAI. ${ }^{3}$ Subdural haemorrhage (SDH) and subarachnoid haemorrhage (SAH) are common abusive injuries. ${ }^{2}$ Shaking of the infant results in angular acceleration and deceleration shear forces that may tear the cortical bridging veins, resulting in $\mathrm{SAH}$ or $\mathrm{SDH} .{ }^{4} \mathrm{SAH}$ may also occur from direct contact forces which cause tearing of vessels. ${ }^{2}$

Although there is no direct relationship between our patient's specific cardiac defect and the physical abuse, a study by Ammerman and Patz indicates that children with disabilities and chronic illnesses may be at higher risk for NAI. ${ }^{5}$ Possible mechanisms for this include overwhelming demands on the parent in caring for the child and disruptions in the attachment process caused by long periods spent in hospital. ${ }^{5}$

\section{Conclusion}

A radiologist should look further than the obvious pathology on a radiograph. When imaging children, radiologists should always look for features of NAI, in particular in children with chronic diseases or disabilities.

1. Ferguson E, Krishnamurthy R, Oldham. Classic imaging signs of congenital cardiovascular abnormalities. Radiographics 2007; 27:1323-1334

2. Lonergan GJ, Baker AM, Morey MK, Boos SC. Child abuse: Radiographic-pathologic correlation. Radiographics 2003; 23:811-845.

3. Offiah A, Van Rijn R, Perez-Rossello JM, Kleinman PK. Skeletal imaging of child abuse (non-accidental injury). Pediatr Radiol 2009; 39:461-470.

4. Tung G, Monica Kumar M, Richardson RC, Jenny C, Brown W. Comparison of accidental and nonaccidental traumatic head injury in children on noncontrast computed tomography. Pediatrics 2006; 118:626-633.

5. Ammerman RT, Patz RJ. Determinants of child abuse potential: Contribution of parent and child factors. J Clin Child Psychol 1996; 25(3):300-307. 